\title{
Filler Network Recovery of Carbon Black Filled Rubber
}

\author{
Yoshinobu ISONO* and Taiki TERASAKI \\ Department of Materials Science and Technology, Nagaoka University of Technology, \\ Nagaoka, Niigata 940-2188, Japan \\ *Corresponding Author: yisono@nagaokaut.ac.jp \\ Received March 10, 2012; Accepted March 30, 2012 \\ (C)2012 The Society of Rubber Industry, Japan
}

\begin{abstract}
Change and restoration in carbon black filler network in SBR through reversing double-step large shear deformations were studied by the use of differential dynamic modulus. Unfilled rubber showed drop and complete recovery in $G^{\prime}$ after elimination of step shear strain $\gamma=0.5$, showing drop in $G^{\prime}$ is not due to chain scission but due to filler network rupture. Filled rubber also shows similar change and recovery in $\mathrm{G}^{\prime}$, but rises over the initial value at long time region. This long time behavior may be due to physical aging. Elimination of the effect of physical aging allowed us to estimate relaxation time in restoration process. It was concluded that (a) differential dynamic modulus measured in recovery process is useful for the characterization of filler network, (b) carbon black filler network ruptured by strain recovers to the original structure by particle diffusion process, and (c) recovery rate of carbon black filler network is independent of amount of filler loading and deformation history.
\end{abstract}

Keywords Rubber, Carbon black, Filler, Network, Dynamic modulus, Shear, Structure Recovery, Relaxation time

\section{Introduction}

Rubber materials filled with nanoparticles such as carbon black show evident nonlinear viscoelastic behaviors such as Payne effect ${ }^{1-5)}$. The nonlinearity observed in unfilled polymer is much weaker than that in filled rubber $^{6-15)}$. For example, unfilled polymers show linear viscoelastic behaviors even at shear strain $\gamma=0.1^{8-13)}$, while filled rubbers show nonlinear viscoelastic behaviors at shear less than $\gamma=0.01^{6,7,14,15)}$. This strain-sensitive nature may be due to filler effect. Recently existence of filler network in filled rubbers has been recognized experimentally not only in macroscopic scale by rheometry in reversing double-step deformations ${ }^{16)}$, and differential dynamic modulus ${ }^{6}$, but also in microscopic scale by in-situ microscopy $^{17,18)}$ and $3 \mathrm{D}-\mathrm{TEM}^{19-22)}$. The knowledge leads us to the idea that the change in carbon black network occurs in large deformations and it leads nonlinearity in viscoelastometry. This plausible idea has been confirmed in electric resistance measurements of filled rubbers ${ }^{6}$ and $3 \mathrm{D}-\mathrm{TEM}$ observation of filled rubbers cured in deformed states $^{23)}$.

Structure of filler network once changed by large deformation should be restored to the original structure by deformation recovery with a relaxation time. In order to discuss filled rubber dynamics, estimate of relaxation time in restoration process is required. Recently we have pro- posed novel method to evaluate filler network by mechanical measurements ${ }^{16}$. Let us allow to explain the method briefly again. For the evaluation of filler network, we need simultaneous attainment of filler network rupture and equilibrium conformation of rubber chain. The latter can be achieved in the recovered state after stress relaxed. The situation can be realized by reversing double-step deformation in shear as well as in compression. Hence possible method may be observation of differential dynamic modulus measured by small oscillatory deformations superposed on large shear and recovery. In addition we have proposed new evaluation method of relaxation time in filler dynamics with the elimination of physical aging effect from recovery curve of differential storage modulus in reversing double-step recoverable deformations ${ }^{16)}$.

The purpose of this work is to study filler network recovery process of carbon black filled rubbers by considering the effect of filler loading, temperature, and magnitude of strain on relaxation time for filler network recovery.

\section{Prediction of characteristics of relaxation time in carbon black filler network}

Relaxation time in restoration process of filler network, $\tau_{r}$, may be based on diffusion of carbon black aggregates. In general, relaxation time means the time during which the gravity center of motion unit migrates in its own size. 
Hence relaxation time of carbon black aggregate can be written by eq. 1 ,

$$
\tau_{r} \sim \frac{R_{\text {aggregate }}^{2}}{D_{\text {aggregate }}}
$$

where $R_{\text {aggregate }}$ and $D_{\text {aggregate }}$ are radius of equivalent cube and diffusion constant of carbon black aggregate, respectively. Diffusion constant $D_{\text {aggregate }}$ can be written as eq. 2 ,

$$
D_{\text {aggregate }} \sim \frac{k_{B} T}{\zeta_{\text {aggregate }}}
$$

where $k_{B}, T$, and $\zeta_{\text {aggregate }}$ denote Boltzman constant, temperature, and friction constant of carbon black aggregate, respectively. By the use of Stokes-Einstein Law, $\zeta_{\text {aggregate }}$ can be written by eq. 3 ,

$$
\zeta_{\text {aggregate }} \sim 6 \pi \eta_{\text {local }} R_{\text {aggregate }}
$$

where $\eta_{\text {local }}$ denotes local viscosity of matrix polymer. Hence $\tau_{r}$ can be shown as eq. 4 .

$$
\tau_{r} \sim \frac{6 \pi \eta_{\text {local }} R_{\text {aggregate }}{ }^{3}}{k_{B} T}
$$

$\eta_{\text {local }}$ and $R_{\text {aggregate }}$ depend on neither amount of carbon black loading nor strain. Therefore characteristics of relaxation time in carbon black filler network $\tau_{r}$ may be predicted as next points.

a) $\tau_{r}$ is independent of amount of carbon black loading.

b) $\tau_{r}$ is independent of strain given to filled rubber.

c) $\tau_{r}$ is proportional to $\mathrm{T}^{-1}$.

\section{Experimental}

\section{Materials}

The rubber used was styrene-butadiene random copolymer (Nipol SBR1502, $\mathrm{M}_{\mathrm{w}}=4.3 \times 10^{5}, \mathrm{~T}_{\mathrm{g}}=-52^{\circ} \mathrm{C}$, Zeon Corporation). Carbon black (CB) used was N330 (SEAST 3 , Tokai Carbon) having average primary particle sizes of $28 \mathrm{~nm}$. Other additives are zinc oxide (Shodo Chemical), Antioxidant, N-(1,3-dimethylbutyl)-N'-phenyl- $p$ phenylenediamine (6PPD, Ouchi Shinko Chemical Industrial), stearic acid (Stearic Acid N for industrial use, Asahi-Denka), sulfur (powdered, 200 mesh, Tsurumi Chemical Industry) and accelerator, N-cyclohexyl-2benzothiazolylsulfenamido (CBS, Ouchi Shinko Chemical Industrial). Details on compound formulation are shown in Table 1. CB master batches are prepared in $1 \mathrm{~L}$ Kneader (DS1-5MHB-S, Moriyama) for $3.5 \mathrm{~min}$. at $343 \mathrm{~K}$ in the beginning (at ca. $390 \mathrm{~K}$ in the final stage, depending on com-

\begin{tabular}{|c|c|c|c|c|}
\hline Sample No. & 1 & 2 & 3 & 4 \\
\hline SBR1502 & 100 & 100 & 100 & 100 \\
\hline CB (N330) & & 20 & 35 & 60 \\
\hline $\mathrm{ZnO}$ & 3 & 3 & 3 & 3 \\
\hline Steraric Acid & 2 & 2 & 2 & 2 \\
\hline Anti-Oxidant ${ }^{\mathrm{a})}$ & 1 & 1 & 1 & 1 \\
\hline Sulfur & 1.5 & 1.5 & 1.5 & 1.5 \\
\hline Accelerator $\mathrm{CBS}^{\text {b) }}$ & 1 & 1 & 1 & 1 \\
\hline
\end{tabular}
pounds). The curatives are mixed to the master batches using 6 inches two-roll mill (Kansai Roll). The compounds
Table 1. Sample formulations (unit in phr).

a) $\mathrm{N}$-(1,3-dimethylbutyl)- $\mathrm{N}^{\prime}$-phenyl-p-phenylenediamine

b) $\mathrm{N}$-Cyclohexyl-2-benzothiazolylsulfenamide

are cured for $30 \mathrm{~min}$. at $433 \mathrm{~K}$ under a pressure of $3 \mathrm{MPa}$.

\section{Measurement}

A specimen used in this work was a disc-shaped samples ( $20 \mathrm{~mm}$ in diameter and $2 \mathrm{~mm}$ in tickness). Both ends of the specimen are fixed to steel plates with cyanoacrylate glue.

The apparatus used for linear and nonlinear viscoelastometry was Multi-Axis/Combined Wave Viscoelasticity Analyzer VR-7310 designed by us (Ueshima Seisakusho).

The measurements of differential dynamic modulus were made in shear deformation mode. Dynamic moduli in linear region of strain were measured with shear strain amplitude of 0.01 for unfilled SBR and that of 0.005 for carbon black filled SBRs at $1 \mathrm{~Hz}$. The dynamic moduli in linear region were used as initial values at equilibrium. Then large step shear strains $0.25,0.5$ or 0.75 were started at time zero. At intervals, small oscillatory shears were imposed around axis. At a time $t_{1}=1800 \mathrm{~s}$, the shear deformation was recovered to zero strain, followed by intermittent measurements of $\mathrm{G}^{*}\left(\omega, \gamma_{L},-\gamma_{L} ; t_{1}, t\right)$ at $1 \mathrm{~Hz}$. Temperature ranged from 40 to $80^{\circ} \mathrm{C}$.

\section{Results}

\section{Unfilled, Cured SBR}

Figure 1 shows the results in shear and recovery experiments for the unfilled, cured sample 1 . The broken line denote the initial value of storage modulus, $\mathrm{G}^{\prime}(\omega, 0)$, measured before imposition of large shear strain. The open and filled symbols show the data in shear and in recovery in shape, respectively. The elimination of large shear was made at time $t_{1}=1800 \mathrm{~s}$. The data of $\mathrm{G}^{\prime}$ show decrease in shear for the sample 1 . In eliminating the shear deformation, however, they recover to the corresponding initial values as expected. This means that the drop in $\mathrm{G}^{\prime}$ after large shearing is not due to chain scission but due to filler network rupture. 


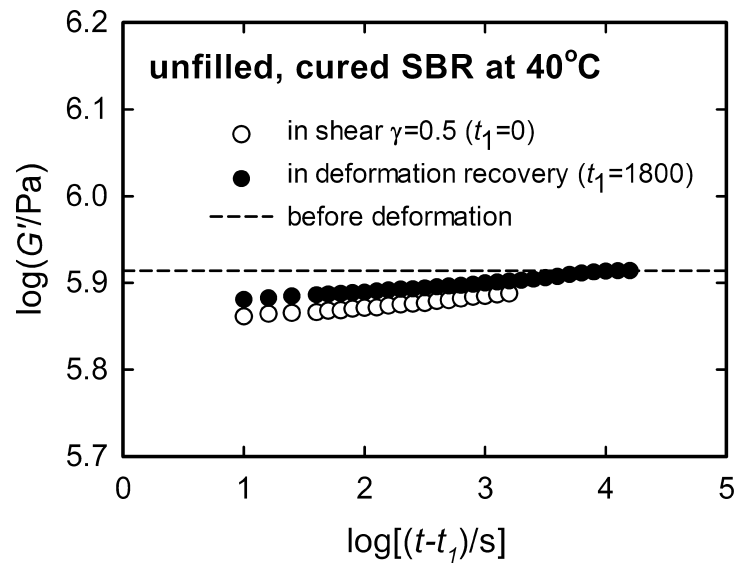

Figure 1. Double-logarithmic plots of differential dynamic modulus against time, $t-t_{1}$, for unfilled, cured sample 1. Open and filled symbols denote the values of $G^{\prime}$ in shear $\gamma=0.5$ and recovered state, respectively. Large shear and reversing shear were given at time $t_{1}=0$ and $t_{1}=1800 \mathrm{~s}$, respectively. The broken line denotes the initial value of $\mathrm{G}^{\prime}$ before straining.
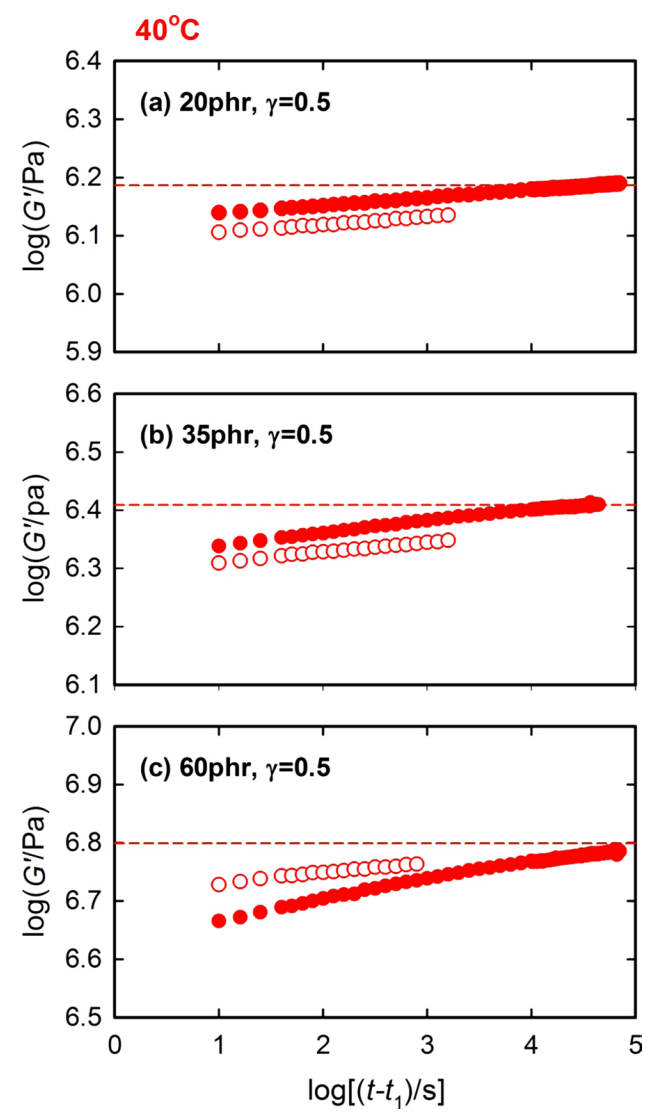

Figure 2. Double-logarithmic plots of differential storage modulus against time for filled, cured samples $2-4$ at $40^{\circ} \mathrm{C}$ and $\gamma=0.5$. The meanings of the symbols and lines are the same as in Figure 1.

\section{Filled, Cured SBRs}

Figures 2-4 show the results for various $\mathrm{CB}$ filled, cured SBRs at temperatures 40,60 , and $80^{\circ} \mathrm{C}$. The meanings of
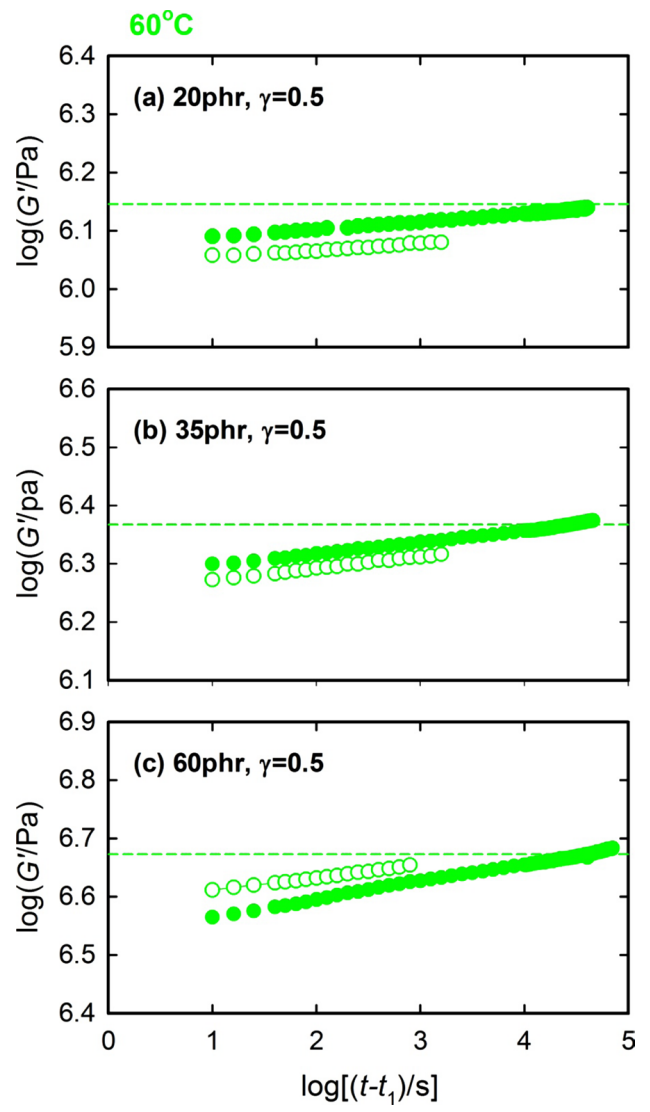

Figure 3. Double-logarithmic plots of differential storage modulus against time for filled, cured samples $2-4$ at $60^{\circ} \mathrm{C}$ and $\gamma=0.5$. The meanings of the symbols and lines are the same as in Figure 1.

the symbols are the same as in Figure 1. In the previous study ${ }^{16)}$, storage modulus and loss tangent showed similar features, although the sign in the deviation was opposite. So the results on $\mathrm{G}^{\prime}$ are shown. Storage modulus $\mathrm{G}^{\prime}$ in shear deformations $(\gamma=0.5)$ show values lower than the initial value, which is similar to the results for unfilled, cured SBR. However, we can find the feature to be different between lower CB concentrations (samples 2 and 3) and higher concentration (sample 4). Samples 2 and 3 shows recovery in $G^{\prime}$ by elimination of step shear strain, but sample 4 shows additional drop just after strain recovery. Same features can be observed irrespective of temperatures. The results show that filler network is easily ruptured in higher carbon black filler concentration. All samples, however, recover to the initial structure as expected. Similar results were obtained for the experiments in different shear strains, $\gamma=0.25$ and 0.75 .

\section{Discussion}

In previous study ${ }^{16)}$, it was pointed out that the $\mathrm{G}^{\prime}$ 

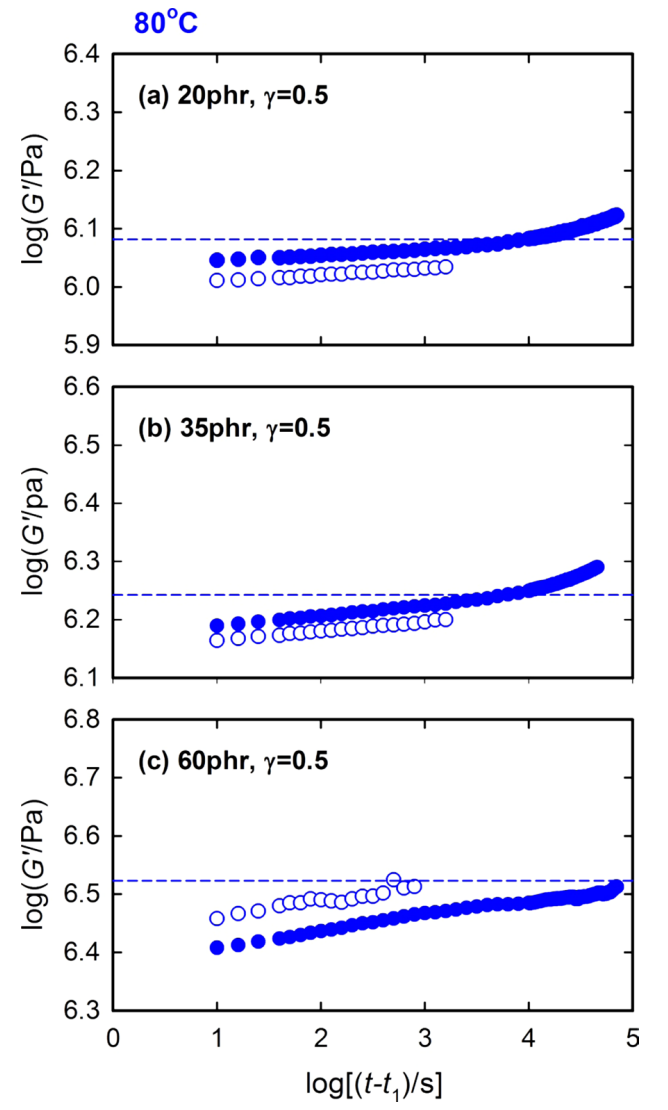

Figure 4. Double-logarithmic plots of differential storage modulus against time for filled, cured samples $2-4$ at $80^{\circ} \mathrm{C}$ and $\gamma=0.5$. The meanings of the symbols and lines are the same as in Figure 1.

curves in restoration process rise over the original values. Similar features can be found in the present results, too. Linear relationships between storage modulus and time elapsed were observed at long time regions as shown in Figure 5. Other samples also showed similar results. The slow increase in $\mathrm{G}^{\prime}$ is considered to be due to physical aging effect of carbon black filled rubber ${ }^{16)}$. The lines in Figures 5(a)-(c) are allowed to be taken by extension of the data points over $20 \mathrm{ks}$ with the use of linear least square regression. If we are allowed to assume that the change in $\mathrm{G}^{\prime}$ due to physical aging are added onto the $\mathrm{G}^{\prime}$ change due to filler network recovery, we may be able to take the plots of the difference between the value of $\mathrm{G}^{\prime}$ of the physical aging line and that observed, $\Delta \mathrm{G}^{\prime} \equiv \mathrm{G}^{\prime}\left(t-t_{1}\right)-\mathrm{G}^{\prime}\left(\omega, \gamma_{L}\right.$, $\left.-\gamma_{L} ; t_{1}, t\right)$, against time elapsed as shown in Figure 6. The curves in Figures 6(a)-(c) show exponential decay at long time region. From the slopes of the lines in Figures 6(a)(c), the longest relaxation time $\tau_{r}$ can be estimated. Similar procedures can be taken for the data in different step shear strains as shown in Figures 7(a)-(c).

The carbon black concentration dependence of the relax-

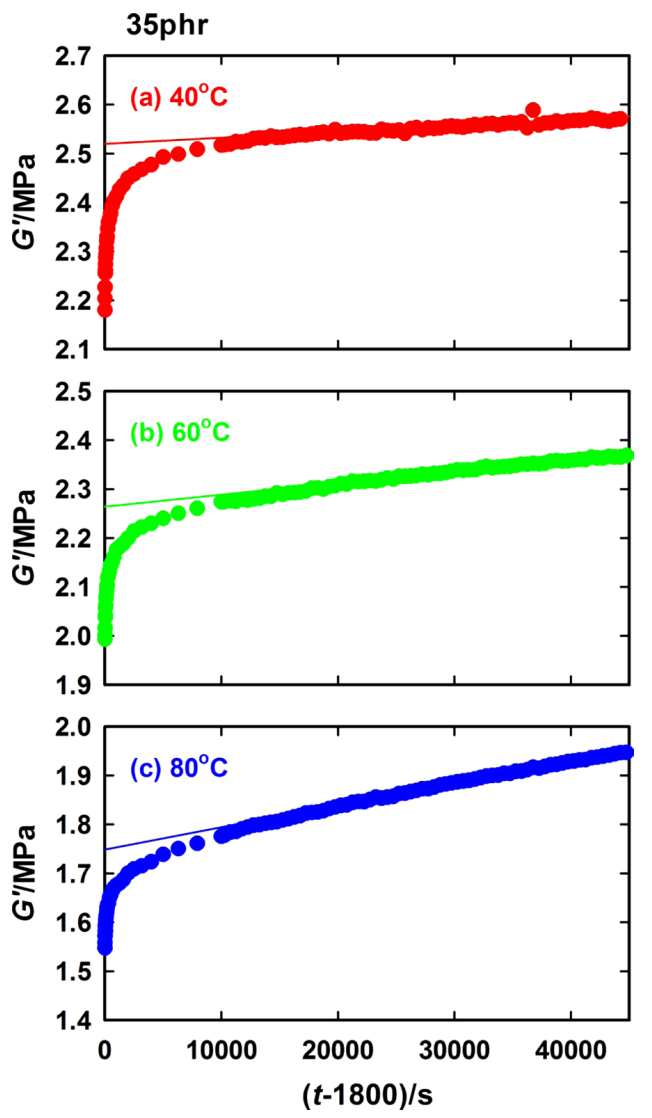

Figure 5. Time dependence of differential storage modulus after deformation recovery for the filled, cured sample 3 at $\mathrm{T}=40-80^{\circ} \mathrm{C}$. The lines in the panels are taken by extension of the data points over $20 \mathrm{ks}$ with the use of linear least square regression.

ation time $\tau_{r}$ is shown in Figure 8, where the values of $\tau_{r}$ at different step shear strain at $40^{\circ} \mathrm{C}$ are also plotted. It can be found clearly that $\tau_{r}$ is independent of amount of carbon black loading and step shear strain, but dependent on $\mathrm{T}^{-1}$. These characteristics agree well with the prediction shown in the Introduction.

We may have next conclusions.

1) Differential dynamic modulus measured in recovery process is useful for the characterization of filler network.

2) Carbon black filler network ruptured by strain recover to the original structure by particle diffusion process.

3) Recovery rate of carbon black filler network is independent of amount of filler loading and deformation history.

\section{Acknowledgemet}

This work was supported in part by Grant-in-Aid for Scientific Research (B) (No. 21360330) from the Ministry of Education, Science, Sports and Culture, Japan. 

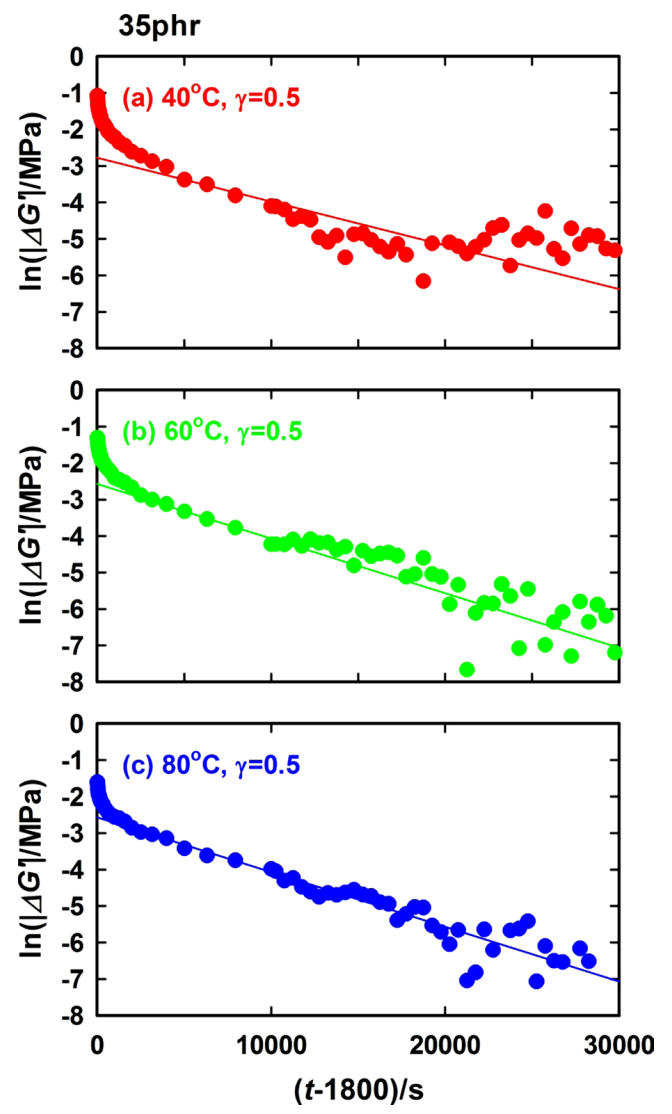

Figure 6. Semi-logarithmic plots of the difference between the value of the line in Figure 5(a)-(c) and that of differential storage modulus after deformation recovery against time after deformation recovery for filled, cured sample 3 .

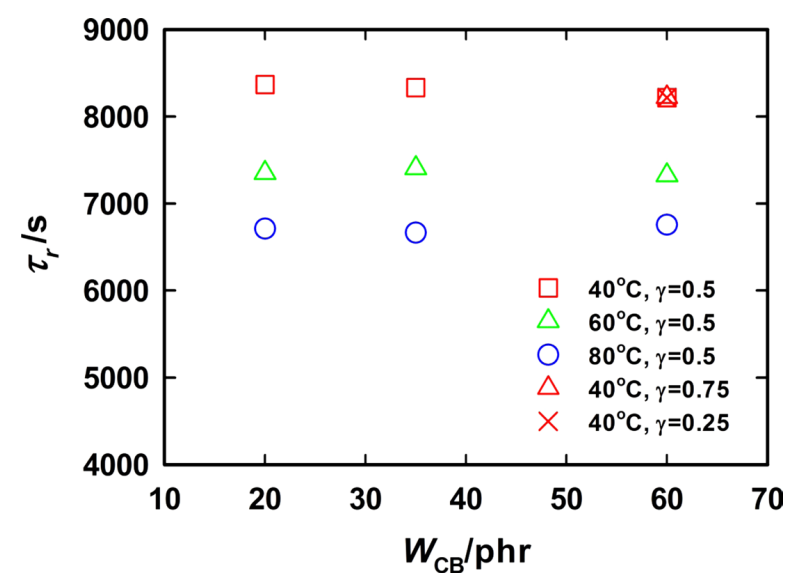

Figure 8. Carbon black concentration dependence of relaxation time estimated for filler network restoration process after deformation recovery at various temperatures and various shear strains.

\section{References}

1) Payne A. R.: J. Appl. Polym. Sci., 6, 57 (1962).

2) Medalia A.: Rubber Chem. Technol., 51, 437 (1978).
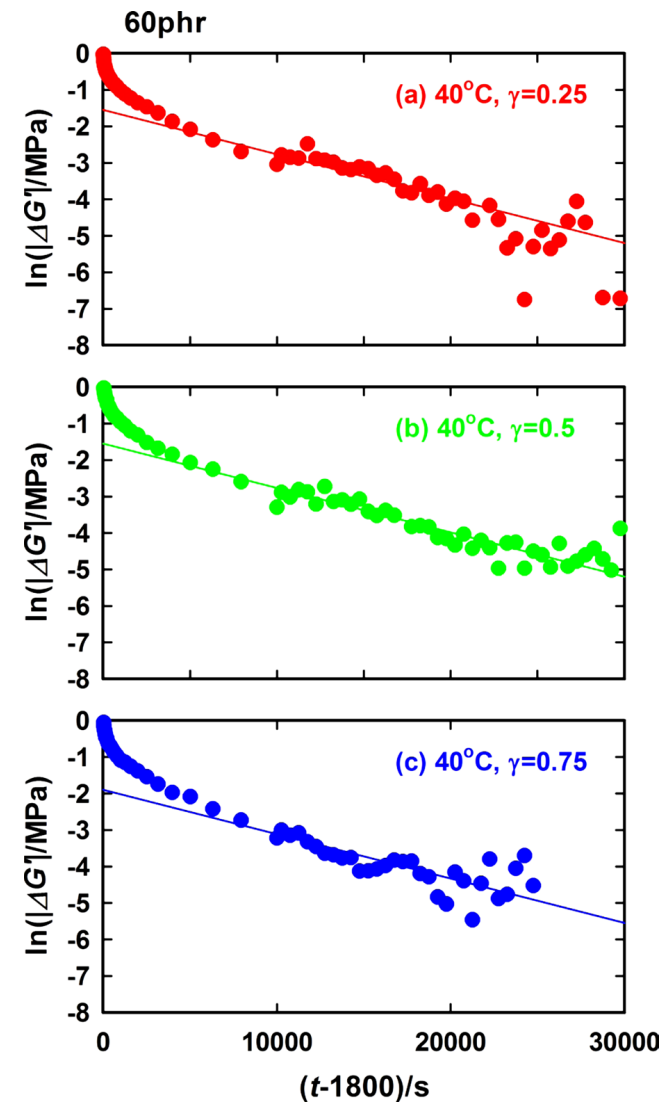

Figure 7. Semi-logarithmic plots similar to Figure 6. Sample is $60 \mathrm{phr}$ filled, cured sample 4 at $40^{\circ} \mathrm{C}$ and $\gamma=0.25-0.75$.

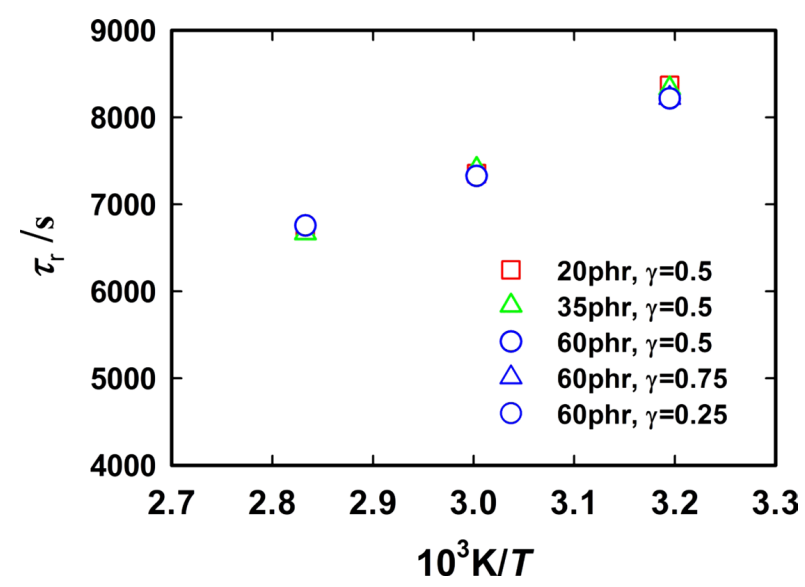

Figure 9. Temperature dependence of relaxation time estimated for filler network restoration process after deformation recovery at various carbon black concentrations and various shear strains.

3) Ferry J. D.: "Viscoelastic Properties of Polymers", 3rd ed., Wiley \& Sons, New York, 1980.

4) Heinrich G., Klueppel M.: Adv. Polym. Sci., 160, 1 (2002).

5) Klueppel M.: Adv. Polym. Sci., 164, 1 (2003).

6) Satoh Y., Fujii S., Kawahara S., Isono Y., Kagami S.: e-J. Soft Mater., 3, 29-40 (2007). 
7) Isono Y., Ferry J. D.: Rubber. Chem. Technol., 57, 925 (1984).

8) Isono Y., Ferry J. D.: J. Rheol., 29, 273 (1985).

9) Isono Y., Ito K., Komiyatani T., Fujimoto T.: Macromolecules, 24, 4429 (1991).

10) Isono Y.: Nippon Gomu Kyokaishi, 67, 89 (1994).

11) Isono Y., Nishitake T.: Polymer, 36, 1635 (1995).

12) Isono Y., Kamohara T., Takano A., Kase T.: Rheol. Acta, 36, 245 (1997).

13) Isono Y., Kawahara S., Kase T.: Nihon Rheoloji Gakkaishi, 31, 201 (2003).

14) Isono Y., Oyama T., Kawahara S.: Adv. Technol. Mater. Mater. Process. J., 5, 84 (2003).

15) Isono Y.: e-J. Soft Mater., 8, 1-7 (2011).

16) Satoh Y., Suda K., Fujii S., Kawahara S., Isono Y., Kagami S.:
$e$-J. Soft Mater., 3, 14 (2007).

17) Suh Y. J., Friedlander S. K.: J. Appl. Phys., 93, 3515 (2003).

18) Rong W., Pelling A. E., Ryan A., Gimzewski J. K., Friedlander S. K.: Nano Letters, 4, 2287 (2004).

19) Ikeda Y., Katoh A., Shimanuki J., Kohjiya S.: Macromolecular Rapid Communications, 25, 1186 (2004).

20) Kohjiya S., Katoh A., Shimanuki J., Hasegawa T., Ikeda Y.: J. Mater. Sci., 40, 2553 (2005).

21) Kohjiya S., Katoh A., Shimanuki J., Hasegawa T., Ikeda Y.: Polymer, 46, 4440 (2005).

22) Kohjiya S., Katoh A., Suda T., Shimanuki J., Ikeda Y.: Polymer, 47, 3298 (2006).

23) Katoh A, Isono Y,: J. Appl. Polym. Sci., DOI: 10.1002/APP. 38356 (in press) 